\title{
Variables contextuales y personales que inciden en el comportamiento violento del niño
}

\author{
Antonia Pelegrín ${ }^{1}$ y Enrique J. Garcés de Los Fayos ${ }^{2}$ \\ ${ }^{1}$ Universidad Miguel Hernández (España) \\ ${ }^{2}$ Universidad de Murcia (España)
}

La conducta agresiva de los niños, niñas y adolescentes se encuentra favorecida por una serie de variables de riesgo, denominadas también variables predictoras. La acumulación de estas variables, a lo largo de la historia de desarrollo del niño, puede predisponerle a un patrón de comportamiento más agresivo que le dificulte la adaptación a su entorno social, familiar y escolar. Las variables implicadas en la manifestación de la conducta agresiva del niño pueden ser múltiples y se clasifican en personales, familiares, escolares y ambientales. Así, nuestro propósito ha sido comprobar la implicación de algunas variables en el desarrollo de un comportamiento más agresivo, violento y potencialmente antisocial y delictivo en los jóvenes. Para ello, los resultados que presentamos, han sido elaborados a partir de un trabajo de investigación más amplio, cuya muestra se encuentra representada por 1.800 alumnos/as de diferentes municipios de la Región de Murcia, con un rango de edad comprendido entre los 11 y 16 años. Entre los resultados más destacados, obtenemos que determinadas variables personales, sociales y educativas, parecen favorecer un comportamiento antisocial y delictivo en los jóvenes.

Palabras clave: Agresión, antisocial, delictivo, variables de riesgo, niños, adolescentes.

Personal and contextual variables that affect the child's violent behaviour. The aggressive behavior of children and adolescents is favored by a number of risk variables, also known as predictive variables. The accumulation of these variables, over the history of child development, may predispose a pattern of behavior more aggressive than it hinders adaptation to their social environment, family and school. The variables involved in the manifestation of the aggressive behavior of children may be manifold and are classified as personal, family, school and environmental issues. Thus, our aim has been to check the involvement of some variables in developing a more aggressive behaviour, potentially violent and antisocial and criminal youth. To that end, we present the results have been produced from a research broader, whose sample is represented by 1800 students from different municipalities of Murcia, with an age range between 11 and 16. Among the most outstanding results, we get that certain variables personal, social and educational, seem to favour antisocial and criminal behavior among young people.

Key words: Aggression, antisocial, criminal, risk variables, children, adolescents.

Correspondencia: Antonia Pelegrín Muñoz. Departamento de Psicología de la Salud, Edificio Altamira. Universidad Miguel Hernández. Avenida de la Universidad, s/n, C.P. 03202, Elche (España). E- mail: apelegrin@umh.es. 
La conducta agresiva de los niños, niñas y adolescentes se encuentra favorecida por una serie de variables de riesgo. La acumulación de estas variables, a lo largo de la historia del desarrollo del niño, puede predisponerle a un patrón de comportamiento más agresivo, que le dificulte la adaptación a su entorno social, familiar y escolar (Loeber, 1990). Las variables implicadas en la manifestación de la conducta agresiva del niño pueden ser múltiples y se clasifican en personales, familiares, escolares y ambientales. No obstante, a pesar de ser diversas las variables predictoras, y ser muchos los estudios que han identificado aquellas causas que preceden al comportamiento agresivo, aún no se tiene suficiente conocimiento de algunas de ellas y otras no se encuentran suficientemente avaladas (Goldstein, 1999; Pelegrín y Garcés de Los Fayos, 2004).

Diversos trabajos (Farrington, 1991; Huesmann y Eron, 1984) han planteado que el comportamiento agresivo se mantiene bastante estable desde la infancia hasta la edad adulta, considerándose esta estabilidad como uno de los factores de riesgo más importantes. También, son diferentes los autores (Achenbach y Edelbrock, 1986; Moffitt, Caspi, Rutter y Silva, 2003; Pelegrín, 2004) que sugieren una mayor tendencia de comportamientos agresivos en los niños respecto a las niñas. Y parece ser, que alrededor de los 13 años, el riesgo de atacar físicamente tiende a ser mayor (Ortega y MoraMerchán, 1997; Pelegrín, 2004). Por otro lado, encontramos otras variables implicadas en la conducta agresiva y violenta del niño, que hacen referencia a características de su personalidad, como un bajo autocontrol (Farrington, 1989), una baja tolerancia a la frustración (Berkowitz, 1969), una baja consideración y respeto hacia los demás (Dodge, Petit, McClaskey y Brown, 1986; Pelegrín, 2004), la inestabilidad emocional (Caprara y Pastorelli, 1996), entre otras. También, las variables familiares tienen especial relevancia. Varios estudios (Prinzie et al., 2004; Stormshak, Bierman, McMahon y Lengua, 2000) han identificado la exposición a estresores familiares (p.Ej., la separación o divorcio de los padres, un clima negativo y conflictivo, o el uso de métodos educativos inadecuados), como factor de riesgo para el desarrollo de problemas de comportamiento en general y, en particular, de la agresión (Bennet y Bates, 1990). Respecto al contexto educativo, otros trabajos (Olweus, 1993; Pelegrín 2004; Trianes, 2000) relacionan el comportamiento agresivo con la conducta bullying y la inadaptación escolar.

Por último, dentro de las variables ambientales, podemos destacar la influencia de los medios de comunicación y el consumo de sustancias nocivas. En este sentido, una de las fuentes principales de la violencia es el modelado simbólico que proporcionan los medios de comunicación, como la televisión (Urra, 1998). Respecto al consumo de 
PELEGRIN y GARCES DE LOS FAYOS. Causas de la violencia infantil

\section{sustancias nocivas, Bushman y Cooper (1990) señalan que existe una relación entre}

Tabla 1. Variables asociadas al comportamiento agresivo y violento

\begin{tabular}{|c|c|}
\hline Grupos & Variables \\
\hline \multicolumn{2}{|l|}{ Personales } \\
\hline & $\begin{array}{l}\text { Sexo } \\
\text { Edad } \\
\text { Variables biológicas } \\
\text { Aparición temprana } \\
\text { Generalización de la situación } \\
\text { Agresión física } \\
\text { Ira } \\
\text { Bajo autocontrol } \\
\text { Impulsividad } \\
\text { Baja tolerancia a la frustración } \\
\text { Egocentrismo/competitividad } \\
\text { Falta de empatía } \\
\text { Distorsión en la percepción de la situación } \\
\text { Locus de control externo } \\
\text { Déficit en habilidades sociales } \\
\text { Sesgos cognitivos } \\
\text { Liderazgo } \\
\text { Baja consideración hacia los demás } \\
\text { Retraimiento social/aislamiento } \\
\text { Inestabilidad emocional } \\
\text { Neuroticismo } \\
\text { Extroversión } \\
\text { Psicoticismo/psicopatía } \\
\text { Desensibilización emocional } \\
\text { Justificación cognitiva } \\
\text { Intencionalidad } \\
\text { Hostilidad } \\
\text { Comparación social } \\
\text { Transferencia de la excitación }\end{array}$ \\
\hline \multicolumn{2}{|l|}{ Familiares } \\
\hline & $\begin{array}{l}\text { Familia } \\
\text { Aprendizaje de comportamientos, creencias y actitudes a través de modelos familiares } \\
\text { Estrés en la familia } \\
\text { Castigo corporal } \\
\text { Reprimendas verbales } \\
\text { Maltrato físico y emocional en la infancia }\end{array}$ \\
\hline \multicolumn{2}{|l|}{ Escolares } \\
\hline & $\begin{array}{l}\text { Conducta "bullying" } \\
\text { Factores internos de la propia institución } \\
\text { Inadaptación escolar } \\
\text { Vigilancia e intervención inadecuada en el lugar de recreo } \\
\text { Influencia y características del grupo de iguales } \\
\text { Rechazo de los iguales } \\
\text { Cambios en la escala de valores }\end{array}$ \\
\hline \multicolumn{2}{|l|}{ Ambientales } \\
\hline & $\begin{array}{l}\text { Video-juegos } \\
\text { Influencia de los medios de comunicación } \\
\text { Nivel socioeconómico } \\
\text { Consumo de sustancias nocivas }\end{array}$ \\
\hline
\end{tabular}


intoxicación alcohólica y comportamiento agresivo, puesto que estos hábitos de consumo supone, en muchas ocasiones, frecuentar ciertos ambientes y adoptar determinadas costumbres de ocio y tiempo libre, que predisponen a la agresión (Elzo, 1996). Así, teniendo en cuenta el gran número de variables que pueden predeterminar dicho comportamiento, en este trabajo hemos analizado algunas de las que han resultado más relevantes en la literatura científica. A continuación, en la Tabla 1, se muestra la relación de variables en sus diferentes contextos (Pelegrín, 2004).

\section{METODO}

\section{Participantes}

La muestra definitiva de estudio estuvo compuesta por un total de 1.800 sujetos escolarizados de diferentes municipios de la Región de Murcia (España). El rango de edad comprende los 11 y 16 años $(M=13,50, D T=1,708)$. Un $49,72 \%$ son hombres $(\mathrm{N}=895)$ y un $50,28 \%$ mujeres $(\mathrm{N}=905)$. La muestra se encuentra distribuida proporcionalmente para cada grupo de edad. El objetivo del que partimos es identificar de entre las variables personales, escolares, sociales y familiares, aquellas que mejor pronostiquen o expliquen la manifestación de un comportamiento agresivo, antisocial y con tendencias delictivas.

\section{Instrumentos}

Cuestionario Sociodemográfico elaborado ad hoc. Se trata de un autocuestionario elaborado por los investigadores, compuesto por con 33 ítems, que recogen información relacionada con características del sujeto (edad, género, curso, número de hermanos, profesión de los padres, entre otras). El formato de respuesta consiste en preguntas abiertas, cerradas con dos opciones de respuesta (sí y no) y tipo Likert de cuatro opciones.

Test Autoevaluativo Multifactorial de Adaptación Infantil (Hernández, $1998,3^{\text {a }}$ ed.). Es un tipo de prueba autoevaluativa que consta de 175 ítems, a los que hay que responder afirmativa o negativamente. Su aplicación puede ser individual o colectiva, desde los 8 a 18 años. El test autoevalúa la Inadaptación Personal, que engloba, tanto el desajuste de las personas que tienen consigo mismas, como con la realidad; la Inadaptación Social, que significa el grado de incapacidad o de problemas que supone la relación social; la Inadaptación Escolar, que evalúa la insatisfacción y el comportamiento inadecuado respecto a la realidad escolar; la Insatisfacción con la Familia, que indica el grado de insatisfacción en cuanto al clima del hogar y la relación de los padres entre sí; la Insatisfacción con los Hermanos, que recoge los conflictos y una relación negativa entre 
los hermanos y, finalmente, las Actitudes Educadoras de los Padres, donde los estilos educadores de los padres son evaluados según el criterio de los hijos. En cuanto a su fiabilidad, se ha utilizado el procedimiento de las dos mitades con la corrección de la fórmula de Sperman-Brown, obteniendo un índice de fiabilidad del 0,87, para el test en general. En relación con los tres niveles estudiados, los resultados son como siguen: Primer Nivel 0,67; Segundo Nivel 0,89 y Tercer Nivel 0,83.

Batería de Socialización-Autoevaluación BAS-3 (Silva y Martorell, 2001, $3^{\text {a }}$ ed.). Se aplica a niños y adolescentes en edades comprendidas entre los 11 y 19 años, aproximadamente. Contiene seis subescalas: Consideración con los demás (Co), que detecta sensibilidad social o preocupación por los demás; Autocontrol en las relaciones sociales (Ac), que representa en su polo positivo acatamiento de reglas y normas sociales $y$, en el polo negativo, conductas agresivas e indisciplina; Retraimiento social (Re), que analiza el apartamiento, tanto positivo como activo, de los demás hasta poder llegar en el extremo a un claro aislamiento; Ansiedad social/Timidez (At), que valora distintas manifestaciones de ansiedad (miedo, nerviosismo) unidas a reacciones de timidez (apocamiento, vergüenza) en las relaciones sociales; Liderazgo (Li), que mide la popularidad, iniciativa y confianza en sí mismo; y, por último, la escala Sinceridad (S). Consideramos conveniente su utilización porque era necesario medir el comportamiento agresivo de los niños desde la perspectiva socializadora. Las escalas de la BAS-3 fueron sometidas a dos estudios de fiabilidad: consistencia interna (coeficiente alfa) y test-retest (con un intervalo próximo a los cuatro meses). Para cada una de las escalas los resultados de la consistencia interna como los del test-retest son los siguientes: Consideración con los demás $(0,82$ y 0,42$)$; Autocontrol social $(0,78$ y 0,66$)$; Retraimiento social $(0,81$ y $0,43)$; Ansiedad social/Timidez $(0,78$ y 0,65$)$; Liderazgo/Autoconfianza $(0,73$ y 0,61$)$ y Sinceridad $(0,60$ y 0,64$)$.

Cuestionario A-D, conductas antisociales y delictivas (Seisdedos, 2000, $4^{\mathrm{a}}$ ed.). Se aplica a niños y adolescentes tanto individual como colectivamente. Mide dos dimensiones: la conducta antisocial y la delictiva. Para comprobar su fiabilidad se utilizó el procedimiento de "las dos mitades" o "pares-impares". Los índices se corrigieron con la formulación de Sperman-Brown. Los resultados, expresados como coeficiente de fiabilidad son los siguientes, para la escala A y D respectivamente. Para los Hombres: 0,86 y 0,86 ; para las mujeres: 0,86 y 0,86 .

\section{Procedimiento}

En primer lugar, fueron seleccionados los centros educativos a visitar, así como, el número de jóvenes que participaron en la muestra definitiva. A continuación, se 
solicitó a la Consejería de Educación y Universidades de la Comunidad Autónoma de la Región de Murcia, el permiso necesario para acceder a los centros educativos, con el fin de obtener un consentimiento informado por parte de los profesores de los centros, así como de los padres de los estudiantes. Posteriormente, se llevó a cabo la administración de los instrumentos de evaluación en las horas de clase de tutoría, según fecha y hora determinado por los colegios. Antes de administrar los cuestionarios, se les explicó detalladamente al grupo de estudiantes en qué consistía el objeto de la investigación, y que los cuestionarios se respondían de forma anónima. El tiempo para responder a los cuestionarios era de una hora. La administración de los cuestionarios se llevó a cabo de forma personal por los Psicólogos expertos en el campo.

La codificación del análisis de los datos se hizo con el programa estadístico SPSS 11.5. Se han realizado análisis descriptivos, reflejándose los porcentajes, las medias y la desviación típica. Por otro lado, se ha llevado a cabo el análisis de Correlación de Pearson y la Regresión Lineal múltiple, utilizando el procedimiento de selección Stepwise o pasos sucesivos.

\section{RESULTADOS}

En este apartado en primer lugar, mostramos un resumen de las variables que han obtenido una significación estadística utilizando la Correlación de Pearson como índice de asociación (Tabla 2). En segundo lugar, se analizarán los resultados obtenidos en el análisis de regresión lineal múltiple utilizando el procedimiento de selección Stepwise o pasos sucesivos. Dicho procedimiento, nos permite identificar, de entre el conjunto de variables disponibles (personales, escolares, sociales y familiares), aquéllas que son capaces de pronosticar o explicar la manifestación de un comportamiento agresivo, antisocial y con tendencias delictivas de la mejor forma posible.

En general, si observamos las matrices de correlaciones, las variables Inadaptación Escolar $(\mathrm{r}=, 532, \mathrm{p}=, 000)$, Inadaptación Social $(\mathrm{r}=, 467, \mathrm{p}=, 000)$, Consideración con los Demás ( $\mathrm{r}=-, 392, \mathrm{p}=, 000)$ y Autocontrol Social $(\mathrm{r}=-, 587, \mathrm{p}=, 000)$, alcanzan un alto índice de correlación para la variable Conducta Antisocial. Para la Conducta Delictiva, los índices de correlación más altos con las subescalas del TAMAI, también se encuentran en la Inadaptación Escolar $(r=, 364, p=, 000)$, y Social $(r=, 402$, $\mathrm{p}=, 000$ ). En las subescalas de la BAS-3, además de obtenerlos con Consideración con los Demás ( $\mathrm{r}=-, 448, \mathrm{p}=, 000)$, y Autocontrol Social $(\mathrm{r}=-, 408, \mathrm{p}=, 000)$, también se obtiene con Retraimiento Social $(\mathrm{r}=, 413, \mathrm{p}=, 000)$. Que el resto de variables muestren significación 
estadística pero con un menor índice de correlación, puede ser debido al amplio tamaño de la muestra.

Tabla 2. Análisis de Correlación de Pearson de las variables que han obtenido significación estadística entre las subescalas del TAMAI, BAS-3 y A-D

\begin{tabular}{|c|c|c|c|}
\hline Subescalas TAMAI & & Antisocial & Delictiva \\
\hline TAMAI-P & $\begin{array}{l}\text { Correlación de Pearson } \\
\text { Sig. (bilateral) } \\
\text { N }\end{array}$ & $\begin{array}{r}, 243 * * \\
, 000 \\
1800\end{array}$ & $\begin{array}{r}, 242 * * \\
, 000 \\
1800\end{array}$ \\
\hline TAMAI-E & $\begin{array}{l}\text { Correlación de Pearson } \\
\text { Sig. (bilateral) } \\
\text { N }\end{array}$ & $\begin{array}{r}, 532 * * \\
, 000 \\
1800\end{array}$ & $\begin{array}{r}, 364 * * \\
, 000 \\
1800\end{array}$ \\
\hline TAMAI-S & $\begin{array}{l}\text { Correlación de Pearson } \\
\text { Sig. (bilateral) } \\
\text { N }\end{array}$ & $\begin{array}{r}, 467 * * \\
, 000 \\
1800 \\
\end{array}$ & $\begin{array}{r}, 402 * * \\
, 000 \\
1800 \\
\end{array}$ \\
\hline TAMAI-F & $\begin{array}{l}\text { Correlación de Pearson } \\
\text { Sig. (bilateral) } \\
\text { N }\end{array}$ & $\begin{array}{r}, 213 * * \\
, 000 \\
1800\end{array}$ & $\begin{array}{r}, 210 * * \\
, 000 \\
1800\end{array}$ \\
\hline TAMAI-H & $\begin{array}{l}\text { Correlación de Pearson } \\
\text { Sig. (bilateral) } \\
\mathrm{N}\end{array}$ & $\begin{array}{r}, 248 * * \\
, 000 \\
1800 \\
\end{array}$ & $\begin{array}{r}, 169 * * \\
, 000 \\
1800 \\
\end{array}$ \\
\hline \multicolumn{4}{|l|}{ Subescalas BAS-3 } \\
\hline Consideración & $\begin{array}{l}\text { Correlación de Pearson } \\
\text { Sig. (bilateral) } \\
\text { N }\end{array}$ & $\begin{array}{r}-, 392 * * \\
, 000 \\
1800\end{array}$ & $\begin{array}{r}-, 408 * * \\
, 000 \\
1800\end{array}$ \\
\hline Autocontrol & $\begin{array}{l}\text { Correlación de Pearson } \\
\text { Sig. (bilateral) } \\
\text { N }\end{array}$ & $\begin{array}{r}-, 587 * * \\
, 000 \\
1800\end{array}$ & $\begin{array}{r}-, 408 * * \\
, 000 \\
1800\end{array}$ \\
\hline Retraimiento & $\begin{array}{l}\text { Correlación de Pearson } \\
\text { Sig. (bilateral) } \\
\text { N }\end{array}$ & $\begin{array}{r}, 281 * * \\
, 000 \\
1800\end{array}$ & $\begin{array}{r}413 * * \\
, 000 \\
1800\end{array}$ \\
\hline Ansiedad & $\begin{array}{l}\text { Correlación de Pearson } \\
\text { Sig. (bilateral) } \\
\text { N }\end{array}$ & $\begin{array}{r}, 036 \\
, 124 \\
1800\end{array}$ & $\begin{array}{r}, 103 * * \\
, 000 \\
1800\end{array}$ \\
\hline
\end{tabular}

** La correlación es significativa al nivel 0,01 (bilateral). TAMAI-P= Inadaptación Personal; TAMAI-E= Inadaptación Escolar; TAMAI-S= Inadaptación Social; TAMAI-F= Insatisfacción Familiar y TAMAI-H= Insatisfacción con los Hermanos.

A continuación, presentamos los resultados obtenidos en el análisis de Regresión Lineal Múltiple (Tablas 3 y 4). Para dicho análisis se ha aplicado el procedimiento de selección por pasos sucesivos (Stepwise). De este modo, podremos comprobar el modelo que mejor explica las variables dependientes: Conducta Antisocial y Conducta Delictiva, tomando como posibles predictores las subescalas del TAMAI y de la BAS-3. 
Los datos recogidos en las tablas anteriores muestran que, de entre las introducidas en el modelo, la Inadaptación Escolar (TAMAI-E) es la que explica la mayor

Tabla 3. Modelo del análisis de regresión lineal múltiple de la variable conducta antisocial y subescalas del TAMAI

\begin{tabular}{cccccccccr}
\hline Modelo & $\mathrm{R}$ & $\mathrm{R}^{2}$ & $\begin{array}{c}\mathrm{R}^{2} \\
\text { corregida }\end{array}$ & Error Típ. & Cambio R & $\begin{array}{c}\text { Cambio en } \\
\mathrm{F}\end{array}$ & $\mathrm{gl1}$ & $\mathrm{g} 12$ & $\mathrm{P}(*)$ \\
\hline 1 &, $532^{\mathrm{a}}$ &, 283 &, 283 & 4,027 &, 283 & 709,471 & 1 & 1798 &, 000 \\
2 &, $580^{\mathrm{b}}$ &, 336 &, 335 & 3,876 &, 053 & 144,173 & 1 & 1797 &, 000 \\
3 &, $582^{\mathrm{c}}$ &, 339 &, 338 & 3,868 &, 003 & 8,248 & 1 & 1796 &, 004 \\
4 &, $585^{\mathrm{d}}$ &, 342 &, 341 & 3,860 &, 003 & 8,094 & 1 & 1795 &, 004 \\
\hline
\end{tabular}

p (*) Significación del cambio en F. Variables predictoras: (Constante), Inadaptación escolar. Variables predictoras: (Constante), Inadaptación escolar, Inadaptación social. Variables predictoras: (Constante), Inadaptación escolar, Inadaptación social, Insatisfacción con el ambiente familiar. Variables predictoras: (Constante), Inadaptación escolar, Inadaptación social, Insatisfacción con el ambiente familiar, Inadaptación personal.

Tabla 4. Coeficientes del análisis de regresión lineal múltiple de la variable conducta antisocial

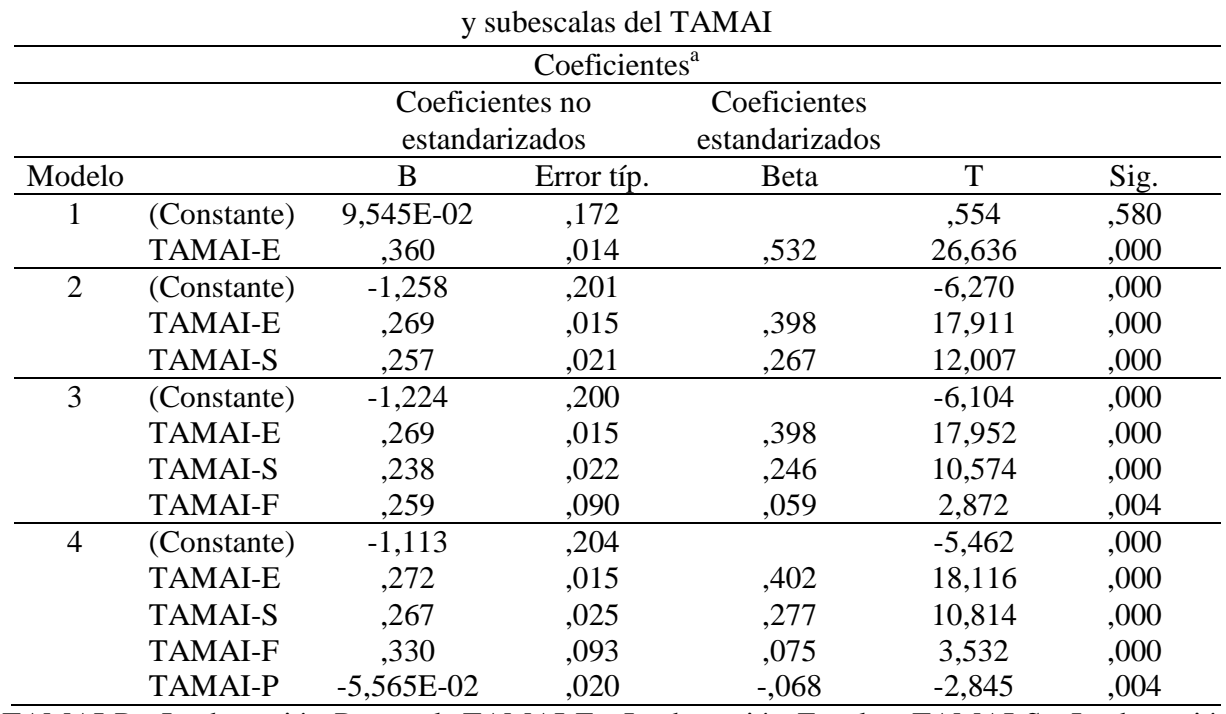

TAMAI-P= Inadaptación Personal; TAMAI-E= Inadaptación Escolar; TAMAI-S= Inadaptación Social; TAMAI-F= Insatisfacción Familiar y TAMAI-H= Insatisfacción con los Hermanos. a. Variable dependiente: Conducta Antisocial.

proporción de la varianza para la Conducta Antisocial. Así, el valor del coeficiente de determinación $\mathrm{R}^{2}=28,3 \%$ (E.T. 4,027) y la significación del contraste $\mathrm{F}$ global de la regresión $(\mathrm{p}=, 000)$, sugieren que gran parte de la variabilidad de la respuesta viene explicada por la Inadaptación Escolar. Además, si observamos los Coeficientes estandarizados Beta $(\beta=, 402)$, comprobamos que dicha Inadaptación Escolar obtiene la mayor capacidad predictiva $(\mathrm{t}=18,116, \mathrm{p}=, 000)$. La aportación en conjunto del resto de las 
variables al modelo es bastante más pequeña (TAMAI-S, TAMAI-F y TAMAI-P), puesto que de un $\mathrm{R}^{2}=28,3 \%$ representado por TAMAI-E tan sólo aumenta a un $\mathrm{R}^{2}=34,2 \%$ (E.T.= 3,860). Por otro lado, la variable Insatisfacción con los Hermanos (TAMAI-H) queda excluida del modelo $(\mathrm{t}=1,482, \mathrm{p}=, 138)$.

Tabla 5. Modelo del análisis de regresión lineal múltiple de la variable conducta delictiva y subescalas del TAMAI

\begin{tabular}{cccccccccc}
\hline Modelo & $\mathrm{R}$ & $\mathrm{R}^{2}$ & $\begin{array}{c}\mathrm{R}^{2} \\
\text { corregida }\end{array}$ & $\begin{array}{c}\text { Error } \\
\text { Típ. }\end{array}$ & $\begin{array}{c}\text { Cambio } \\
\mathrm{R}^{2}\end{array}$ & $\begin{array}{c}\text { Cambio en } \\
\mathrm{F}\end{array}$ & $\mathrm{gl1}$ & $\mathrm{g} 12$ & $\mathrm{p}(*)$ \\
\hline 1 &, $402^{\mathrm{a}}$ &, 162 &, 162 & 3,349 &, 162 & 347,506 & 1 & 1798 &, 000 \\
2 &, $444^{\mathrm{b}}$ &, 197 &, 196 & 3,279 &, 035 & 78,024 & 1 & 1797 &, 000 \\
3 &, $459^{\mathrm{c}}$ &, 210 &, 209 & 3,253 &, 013 & 30,607 & 1 & 1796 &, 000 \\
\hline
\end{tabular}

p (*) Significación del cambio en F. Variables predictoras: (Constante), Inadaptación social. Variables predictoras: (Constante), Inadaptación social, Inadaptación escolar. Variables predictoras:

(Constante), Inadaptación social, Inadaptación escolar, Insatisfacción con el ambiente familiar.

Por otro lado, de las tres variables introducidas en el modelo (TAMAI-S, TAMAI-E y TAMAI-F), la Inadaptación Social (TAMAI-S) es la que alcanza un valor más alto en cuanto a la proporción explicada de la varianza para la Conducta Delictiva $\left(\mathrm{R}^{2}=16,2 \%, \mathrm{p}=, 000\right)$. La aportación conjunta de las tres variables introducidas (TAMAI-S, TAMAI-E y TAMAI-F) eleva la $\mathrm{R}^{2}$ a un $21,0 \%$, esto es, un $4,8 \%$ más de

Tabla 6. Coeficientes del análisis de regresión lineal múltiple de la variable conducta delictiva

\begin{tabular}{|c|c|c|c|c|c|c|}
\hline \multicolumn{7}{|c|}{ Coeficientes $^{\mathrm{a}}$} \\
\hline & & \multicolumn{2}{|c|}{$\begin{array}{l}\text { Coeficientes no } \\
\text { estandarizados }\end{array}$} & \multirow{2}{*}{$\begin{array}{c}\begin{array}{c}\text { Coeficientes } \\
\text { estandarizados }\end{array} \\
\text { Beta } \\
\end{array}$} & \multirow[b]{2}{*}{$\mathrm{t}$} & \multirow[b]{2}{*}{ Sig. } \\
\hline Modelo & & $\mathrm{B}$ & Error típ. & & & \\
\hline \multirow[t]{2}{*}{1} & (Constante) & $-1,116$ &, 164 & & $-6,787$ &, 000 \\
\hline & TAMAI-S & ,299 &, 016 & ,402 & 18,642 &, 000 \\
\hline \multirow[t]{3}{*}{2} & (Constante) & $-1,589$ & , 170 & & $-9,362$ & ,000 \\
\hline & TAMAI-S &, 218 &, 018 & ,294 & 12,032 &, 000 \\
\hline & TAMAI-E &, 112 &, 013 & ,216 & 8,833 &, 000 \\
\hline \multirow[t]{4}{*}{3} & (Constante) & $-1,534$ & ,169 & & $-9,099$ & ,000 \\
\hline & TAMAI-S &, 186 & 019 & ,251 & 9,854 &, 000 \\
\hline & TAMAI-E &, 112 & ,013 & ,216 & 8,914 &, 000 \\
\hline & TAMAI-F & 419 &, 076 &, 124 & 5,532 &, 000 \\
\hline
\end{tabular}

Variable dependiente: Conducta delictiva. TAMAI-E= Inadaptación Escolar; TAMAI-S= Inadaptación Social; TAMAI-F= Insatisfacción Familiar y TAMAI-H= Insatisfacción con los Hermanos.

proporción explicada a la variable dependiente. Por tanto, la Inadaptación Social es el predictor con una mayor potencia explicativa sobre la Conducta Delictiva ( $\beta=, 251$, $\mathrm{t}=9,854, \mathrm{p}=, 000)$. La Inadaptación Personal $(\mathrm{t}=-, 879, \mathrm{p}=, 380)$ y la Insatisfacción con los Hermanos $(\mathrm{t}=, 188, \mathrm{p}=, 851)$ quedan excluidas como variables en el modelo (Tablas 5 y $6)$. 
Tabla 7. Modelo del análisis de regresión lineal múltiple de la variable conducta antisocial y subescalas de la BAS-3

\begin{tabular}{|c|c|c|c|c|c|c|c|c|}
\hline Modelo & $\mathrm{R}$ & $\mathrm{R}$ cuadrado & $\begin{array}{l}\mathrm{R} \text { cuadrado } \\
\text { corregida }\end{array}$ & $\begin{array}{l}\text { Cambio R } \\
\text { cuadrado }\end{array}$ & Cambio en $\mathrm{F}$ & gl1 & $\mathrm{gl} 2$ & $\mathrm{p}(*)$ \\
\hline 1 &, $587^{\mathrm{a}}$ &, 345 &, 344 & ,345 & 945,391 & 1 & 1798 & 000 \\
\hline 2 &, $606^{\mathrm{b}}$ &, 367 & ,367 & 023 & 64,964 & 1 & 1797 &, 000 \\
\hline 3 &, $610^{\mathrm{c}}$ & ,372 &, 371 & ,005 & 12,985 & 1 & 1796 & 000 \\
\hline 4 &, $612^{\mathrm{d}}$ &, 375 & ,373 & ,003 & 7,412 & 1 & 1795 & 007 \\
\hline 5 &, $613^{\mathrm{e}}$ & 376 &, 374 & ,002 & 4,353 & 1 & 1794 & 037 \\
\hline
\end{tabular}

p (*) Significación del cambio en F. Variables predictoras: (Constante), Autocontrol. Variables predictoras: (Constante), Autocontrol, Consideración con los demás. Variables predictoras: (Constante), Autocontrol, Consideración con los demás, Liderazgo/Autoconfianza. Variables predictoras: (Constante), Autocontrol, Consideración con los demás, Liderazgo/Autoconfianza, Retraimiento social. Variables predictoras: (Constante), Autocontrol, Consideración con los demás, Liderazgo/Autoconfianza, Retraimiento social, Ansiedad/Timidez.

En este análisis de regresión lineal múltiple, de nuevo hemos utilizado el procedimiento de selección de pasos sucesivos (Stepwise) entre las subescalas de la BAS-3 y la variable dependiente Conducta Antisocial. Así, si observamos los datos de la tabla 7, comprobamos que la variable Autocontrol Social es la que adquiere una mayor capacidad predictiva, puesto que su $\mathrm{R}^{2}$, representa un $34,5 \%$ (E.T. $3,850, \mathrm{p}=, 000$ ) de varianza explicada sobre la variable dependiente Conducta Antisocial. Por otro lado, al introducir los cinco predictores en el modelo, el $\mathrm{R}^{2}$ alcanza un 37,6\% (E.T. 3,761, $\mathrm{p}=, 037$ ); es decir, la proporción de la varianza explicada sobre la variable dependiente Conducta Antisocial, tan sólo aumenta en un 3,0\%. En este sentido, podemos concluir que la variable Autocontrol Social es la que aporta una mayor capacidad predictiva al modelo $(\beta=-, 508, \mathrm{t}=-24,256, \mathrm{p}=, 000)$, quedando excluida la variable Ansiedad Social/Timidez $(\mathrm{t}=-2,086, \mathrm{p}=, 037)($ Tabla 8$)$.

Finalmente, según los resultados de los análisis realizados, quedan cuatro variables incluidas en el modelo seleccionado (Consideración, Autocontrol, Retraimiento Social y Liderazgo/Autoconfianza) para explicar la variable dependiente Conducta Delictiva. De estas cuatro variables, la Consideración con los Demás, es la que mayor proporción de varianza explica sobre dicha variable dependiente Conducta Delictiva con un $\mathrm{R}^{2}$ del 20,1\% (E.T. 3,271, $\mathrm{p}=, 000$ ). Por otro lado, se observa que un $11,6 \%$ $\left(\mathrm{R}^{2}\right.$ corregido $=31,7 \%$, E.T. 3,026, $\left.\mathrm{p}=, 000\right)$ es la aportación conjunta de las tres variables restantes al modelo (Autocontrol Social, Retraimiento Social y Liderazgo/Autoconfianza). En este sentido, la variable Consideración con los Demás, es el mejor predictor de la variable dependiente Conducta Delictiva $(\beta=-, 265, \mathrm{t}=-11,117$, $\mathrm{p}=, 000$ ). La variable Ansiedad Social/Timidez ha sido excluida del modelo al no aportar nada $(\mathrm{t}=-, 502, \mathrm{p}=, 616)$, como muestran las tablas 9 y 10 . 
Tabla 8. Coeficientes del análisis de regresión lineal múltiple de la variable conducta antisocial y subescalas de la BAS-3

\begin{tabular}{|c|c|c|c|c|c|c|}
\hline \multicolumn{7}{|c|}{ Coeficientes $^{\mathrm{a}}$} \\
\hline & & \multicolumn{2}{|c|}{$\begin{array}{l}\text { Coeficientes no } \\
\text { estandarizados }\end{array}$} & \multirow{2}{*}{$\begin{array}{c}\begin{array}{c}\text { Coeficientes } \\
\text { estandarizados }\end{array} \\
\text { Beta }\end{array}$} & \multirow[b]{2}{*}{$\mathrm{t}$} & \multirow[b]{2}{*}{ Sig. } \\
\hline Modelo & & B & Error típ. & & & \\
\hline \multirow[t]{2}{*}{1} & (Constante) & 13,183 &, 164 & & $-6,787$ &, 000 \\
\hline & Autocontrol &,- 943 & ,016 & ,402 & 18,642 &, 000 \\
\hline \multirow[t]{3}{*}{2} & (Constante) & 15,515 &, 170 & & $-9,362$ &, 000 \\
\hline & Autocontrol &,- 825 & ,018 & ,294 & 12,032 &, 000 \\
\hline & Consideración &,- 295 & ,013 & ,216 & 8,833 &, 000 \\
\hline \multirow[t]{4}{*}{3} & (Constante) & 15,023 & , 169 & & $-9,099$ &, 000 \\
\hline & Autocontrol &,- 829 & 019 &, 251 & 9,854 & 000 \\
\hline & Consideración &,- 319 & 013 & ,216 & 8,914 &, 000 \\
\hline & Liderazgo &, 136 &, 076 &, 124 & 5,532 &, 000 \\
\hline \multirow[t]{5}{*}{4} & (Constante) & 14,141 & ,549 & & 25,779 & ,000 \\
\hline & Autocontrol &,- 817 & 034 &,- 508 & $-24,255$ &, 000 \\
\hline & Consideración &,- 278 & 040 &,- 159 & $-6,960$ &, 000 \\
\hline & Liderazgo &, 141 & 038 &, 072 & 3,759 &, 000 \\
\hline & Retraimiento &, 106 &, 039 &, 058 & 2,722 & 007 \\
\hline \multirow[t]{6}{*}{5} & (Constante) & 14,315 & ,554 & & 25,823 & ,000 \\
\hline & Autocontrol &,- 816 & 034 &,- 508 & $-24,256$ &, 000 \\
\hline & Consideración &,- 265 & 040 &,- 151 & $-6,565$ &, 000 \\
\hline & Liderazgo &, 121 & 039 &, 062 & 3,129 & 002 \\
\hline & Retraimiento &, 145 & 043 & ,079 & 3,356 & 001 \\
\hline & Ansiedad & $-7,421 \mathrm{E}-02$ & 036 &,- 045 & $-2,086$ & 037 \\
\hline
\end{tabular}

a. Variable dependiente: Conducta antisocial.

Tabla 9. Modelo del análisis de regresión lineal múltiple de la variable conducta delictiva y subescalas de la BAS-3

\begin{tabular}{|c|c|c|c|c|c|c|c|c|}
\hline Modelo & $\mathrm{R}$ & $\mathrm{R}$ cuadrado & $\begin{array}{c}\text { R cuadrado } \\
\text { corregida }\end{array}$ & $\begin{array}{l}\text { Cambio R } \\
\text { cuadrado }\end{array}$ & Cambio en F & gl1 & Gl2 & $\mathrm{p}(*)$ \\
\hline 1 &, $448^{\mathrm{a}}$ & ,201 & ,200 & ,201 & 451,043 & 1 & 1798 & ,000 \\
\hline 2 &, $506^{\mathrm{b}}$ & ,256 & ,256 & ,056 & 135,098 & 1 & 1797 & ,000 \\
\hline 3 &, $544^{\mathrm{c}}$ & 296 & 295 & ,040 & 101,393 & 1 & 1796 & ,000 \\
\hline 4 &, $563^{\mathrm{d}}$ & ,317 & ,315 & ,021 & 53,863 & 1 & 1795 & ,000 \\
\hline
\end{tabular}

p (*) Significación del cambio en F. Variables predictoras: (Constante), Consideración con los demás. Variables predictoras: (Constante), Consideración con los demás, Autocontrol. Variables predictoras: (Constante), Consideración con los demás, Autocontrol, Retraimiento social. Variables predictoras: (Constante), Consideración con los demás, Autocontrol, Retraimiento social, Liderazgo/Autoconfianza.

\section{DISCUSION}

Para la realización de este trabajo, se parte de la revisión de un amplio número de trabajos realizados, durante los últimos años, acerca del comportamiento agresivo y violento en niños, adolescentes y adultos. Diversos autores (Bandura, 1973; Berkowitz, 1996; McCord, 2000) han analizado, desde diferentes teorías y modelos, las posibles variables de riesgo que pueden originar dicho comportamiento. Así, nuestro propósito ha 
sido comprobar la implicación de algunas variables en el desarrollo de un comportamiento más agresivo, violento y potencialmente antisocial y delictivo en los jóvenes. Una vez situados en el propósito de este trabajo, a continuación presentamos las conclusiones de los resultados más significativos obtenidos.

Tabla 10. Coeficientes del análisis de regresión lineal múltiple de la variable conducta delictiva y subescalas de la BAS-3

\begin{tabular}{|c|c|c|c|c|c|c|}
\hline \multicolumn{7}{|c|}{ Coeficientes $^{\mathrm{a}}$} \\
\hline & & \multicolumn{2}{|c|}{$\begin{array}{l}\text { Coeficientes no } \\
\text { estandarizados }\end{array}$} & $\begin{array}{l}\text { Coeficientes } \\
\text { estandarizados }\end{array}$ & & \\
\hline Modelo & & $\mathrm{B}$ & Error típ. & Beta & $\mathrm{t}$ & Sig. \\
\hline \multirow[t]{2}{*}{1} & (Constante) & 8,724 &, 345 & & 25,257 &, 000 \\
\hline & Consideración &,- 605 & 028 &,- 448 & $-21,238$ &, 000 \\
\hline \multirow[t]{3}{*}{2} & (Constante) & 10,078 &, 353 & & 28,551 &, 000 \\
\hline & Consideración &,- 450 &, 031 &,- 333 & $-14,726$ & 000 \\
\hline & Autocontrol &,- 325 &, 028 &,- 263 & $-11,623$ &, 000 \\
\hline \multirow[t]{4}{*}{3} & (Constante) & 7,484 & 429 & & 17,429 &, 000 \\
\hline & Consideración &,- 323 & 032 &,- 239 & $-10,008$ &, 000 \\
\hline & Autocontrol &,- 287 & 027 &,- 232 & $-10,455$ & ,000 \\
\hline & Retraimiento & 320 & 032 & 228 & 10,069 & , 000 \\
\hline \multirow[t]{5}{*}{4} & (Constante) & 6,573 & 441 & & 14,902 &, 000 \\
\hline & Consideración &,- 358 & 032 &,- 265 & $-11,117$ &, 000 \\
\hline & Autocontrol &,- 292 & 027 &,- 236 & $-10,774$ &, 000 \\
\hline & Retraimiento &, 333 & 031 & 237 & 10,617 & ,000 \\
\hline & Liderazgo & 222 & 030 & 147 & 7,339 & , 000 \\
\hline
\end{tabular}

Variable dependiente: Conducta delictiva.

De este modo, de las variables analizadas destacamos que, aquellos niños más inadaptados escolarmente y con bajo autocontrol en las relaciones sociales, tienen un mayor riesgo en desarrollar una conducta antisocial, lo que les puede hacer más vulnerables a mostrar actitudes agresivas, indisciplinadas y violentas en determinadas situaciones. También, al encontrarse más inadaptados en el centro escolar, pueden manifestar comportamientos agresivos y disruptivos en el aula, así como actitudes desfavorables hacia el aprendizaje, hacia los profesores y hacia la propia institución. Desde otros trabajos, se comprueba cómo se relaciona positivamente la inadaptación escolar con el comportamiento agresivo y violento (DuRant, Candenhead; Pendergrast, Slavens y Linder, 1994). Al parecer, los problemas escolares como las bajas notas, un pobre rendimiento escolar, repetir curso, ser expulsado y las dificultades de aprendizaje (Buka y Earls, 1993), encuentran un vínculo con la conducta problemática y agresiva dentro del aula (Trianes, 2000). Además, dicha conducta dentro del aula, podría estar relacionado con el adolescente bullying puesto que, tanto la conducta bullying como la agresión física en el colegio, son predictores de actos antisociales, de delincuencia y de violencia (Olweus, 1991). 
Por otro lado, un bajo autocontrol puede llevar a responder impulsivamente y con actitud agresiva ante un determinado conflicto. Farrington (1989) encontró que la impulsividad era uno de los seis predictores más importantes de la agresión en niños, entre las edades de 8 a 10 años. Además, la impulsividad es un criterio clave para el diagnóstico del problema de personalidad antisocial y se relaciona con inadaptación posterior y delincuencia (DSM-IV-R, 2002). Estos resultados, en conjunto, aumentan la posibilidad de que quienes sean impulsivos también se muestren más agresivos físicamente en una situación determinada (Halperin et al., 1995). Estos datos son, tal vez, consistentes con otros estudios que indican que la impulsividad se asocia con algunas formas de violencia y delincuencia en la edad adulta (Loeber et al., 1993). No obstante, serían necesarios estudios longitudinales para determinar si las puntuaciones de la impulsividad son predictoras de los trastornos disruptivos del comportamiento (Halperin et al., 1995).

Por último, en cuanto a la conducta delictiva, los niños/as tienen un mayor riesgo a desarrollarla cuando se encuentran más inadaptados socialmente y manifiestan un comportamiento menos considerado hacia sus iguales. Este perfil, puede fomentar comportamientos inadecuados como la falta de respeto, rebeldía hacia las normas establecidas, expresar una mayor agresividad social, enfrentamiento con las personas, desconfiar más y reducir su relación con los iguales (Eisenberg, Fabes, y Losoya, 1997).

\section{AGRADECIMIENTOS}

Este trabajo es complementario de una línea de investigación financiada por el Centro de Alto Rendimiento y de Investigación en Ciencias del Deporte del Consejo Superior de Deportes, con Referencia Número: 07/UPB20/01. La muestra utilizada es la base de dicha línea financiada.

\section{REFERENCIAS}

Achenbach, T.M. y Edelbrock, C. (1986). Manual for the Teachers Report Form and teacher version of the Child Behavior Prolile. Burlington, VT: University of Vermont, Department of Psychiatry.

Bandura, A. (1973). Aggression: A social learning analysis. Englewood Cliffs, NJ: Prentice-Hall.

Bennett, D.S. y Bates, J.E. (1990, May). Attributional style, life stress, and social support as predictors of depressive symptoms and aggressive behaviors in early 
adolescence. Paper presented at the annual meeting of the Midwestern Psychological Asociation. Chicago.

Berkowitz, L. (1969). The frustration-aggression hypothesis revisited. En L. Berkowitz (Ed.), Roots of Aggression. Nueva York: Atherton Press.

Berkowitz, L. (1996). Agresión: causas, consecuencias y control. Bilbao: Desclée de Brouwer.

Buka, S. y Earls, F. (1993). Determinants of delinquency and violence. Health Affair, 12, 46-64.

Bushman, B.J. y Cooper, H. (1990). Effects of alcohol on human aggression: An intergrative research review. Psychological Bulletin, 107, 341-354.

Caprara, G.V. y Pastorelli, C. (1996). Indicadores precoces de la adaptación social. En J. Buendía (Ed.), Psicopatología en niños y adolescentes. Desarrollos actuales. Madrid: Pirámide.

Dodge, K.A., Pettit, G.S., McClaskey, C.L. y Brown, M. (1986). Social competence in children. Monographs of the Society for Research in Child Development, 51, 1-85.

Durant, R.H.; Candenhead, C.; Pendergrast, R.A.; Slavens, G. y Linder, C.W. (1994). Factors associated with the use of violence among urban black adolescents. American Journal of Public Health, 84, 612-617.

Eisenberg, N., Fabes, R.A. y Losoya, S. (1997). Emotional responding: regulation, social correlates and socialization. En P. Salovey y D.J. Sluyter (Eds.), Emotional development and emotional intelligence (pp. 129-163). New York: BasicBooks.

Elzo, J. (1996). Manifestaciones y raíces de la violencia juvenil hoy. Educadores, 38, 180.

Farrington, D.P. (1989). Early predictors of adolescent aggression and adult violence. Violence and Victims, 4, 79-100.

Farrington, D.P. (1991). Childhood aggression and adult violence: Early precursors and later life outcomes. En D.J. Pepler y K.H. Rubin (Eds.), The development and treatment of childhood aggression (pp. 5-29). Hillsdale, NJ: Erlbaum.

Goldstein, A.P. (1999). Aggression reduction strategies: Effective and ineffective. School Psychology Quarterly, 14, 40-58.

Halperin, J.M., Newcorn, J.H., Matier, K., Bedi, G., Hall, S. y Sharma, V. (1995). Impulsivity and the initiation of fights in children with disruptive behavior disorders. Journal Child Psychiatry, 36, 1199-1211.

Hernández, P. (1998). Test Autoevaluativo Multifactorial de Adaptación Infantil: TAMAI. Madrid: TEA.

Huesmann, L.R. y Eron, L.D. (1984). Cognitive processes and the persistence of aggressive behavior. Aggressive Behavior, 10, 243-251. 
Loeber, R. (1990). Development and risk factors of juvenile antisocial behavior and delinquency. Clinical Psychology Review, 10, 1-42.

Loeber, R., Wung, P., Keenan, K., Giroux, B., Stouthamer-Loeber, M. y Van Kammen, W.B. (1993). Developmental pathways in disruptive child behavior. Developmental and Phychopathology, 5, 101-132.

Manual diagnóstico y estadístico de los trastornos mentales. Texto revisado, DSM-IV-TR (2002). Barcelona: MASSON.

McCord, J. (2000). Contribuciones psicosociales a la violencia y la psicopatía. En A. Raine y J. Sanmartín (Eds.), Violencia y psicopatía (pp. 89-130). Barcelona: Ariel.

Moffitt, W., Caspi, A., Rutter, M. y Silva, X. (2003). Sex differences in antisocial behaviour. International Journal of Behavioral Developmental, 27, 190-191.

Olweus, D. (1991). Bully/victim problems among school children: Some basic facts and effects of a school-based intervention program. En D.J. Pepler y K.H. Rubin (Eds.), The Development and treatment of childhood aggression (pp. 411-448). Hillsdale, NJ: Lawrence Erlbaum.

Olweus, D. (1993). Bullying at school: What we know and what we can do. Oxford: Blackwell.

Ortega, R. y Mora-Merchán, J. (1997). Agresividad y violencia. El problema de la victimización entre escolares. Revista de Educación, 313, 7-27.

Pelegrín, A. (2004). El comportamiento agresivo y violento: Factores de riesgo y protección como mediadores de inadaptaciones y adaptaciones en la socialización del niño y el adolescente. Tesis Doctoral no publicada. Murcia: Universidad de Murcia.

Pelegrín, A. y Garcés de Los Fayos, E.J. (2004). Aproximación teórico-descriptiva de la violencia de género: propuesta para la prevención. Apuntes de Psicología, 22, 353-373.

Prinzie, P., Onghena, P., Hellinckx, W., Grietens, H., Ghesquiere, P., Colpin, H. y Prinzie, P. (2004). Parent and Child Personality Characteristics as Predictors of Negative Discipline and Externalizing Problem Behaviour in Children. European Journal of Personality, 18, 73-102.

Seisdedos, N. (2000). Cuestionario A-D, Conductas Antisociales-Delictivas. Madrid: TEA Ediciones.

Silva, F. y Martorell, C. (2001). BAS-3: Batería de Socialización (Autoevaluación). Madrid: TEA.

Stormshak, E.A., Bierman, K.L., McMahon, R.J. y Lengua, L.J. (2000). Parenting practices and child disruptive behavior problems in early elementary school. Journal of Clinical Child Psychology, 29, 17-29.

Trianes, M.V. (2000). La violencia en contextos escolares. Málaga: Aljibe. 
PELEGRIN y GARCES DE LOS FAYOS. Causas de la violencia infantil

Urra, J. (1998). Violencia y medios de comunicación. En J. Sanmartín, J.S. Grisolía y S. Grisolía (Eds.), Violencia, televisión y cine (pp.133-145). Barcelona: Ariel.

Recibido: 8 de octubre de 2007 Modificaciones: 20 de diciembre de 2007 Aceptado: 10 de enero de 2008 